\title{
Surveillance Systems to Track Progress Toward Polio Eradication - Worldwide, 2014-2015
}

Cynthia J. Snider, $\mathrm{PhD}^{1}$; Ousmane M. Diop, $\mathrm{PhD}^{2}$; Cara C. Burns, $\mathrm{PhD}^{3}$; Rudolph H. Tangermann, $\mathrm{MD}^{2}$; Steven G.F. Wassilak, MD ${ }^{1}$

Global efforts to eradicate polio began in 1988, and poliofree certification has been achieved in four of the six World Health Organization (WHO) regions. Nigeria was removed from WHO's list of countries with endemic polio in September 2015, achieving an important milestone toward interruption of wild poliovirus (WPV) transmission in the African Region (1). Afghanistan and Pakistan, both in the Eastern Mediterranean Region, were the only countries to report WPV cases in 2015. Previously reported outbreaks caused by WPV importation during 2013-2014 have ended (2,3). The primary means for detecting poliovirus transmission is surveillance for acute flaccid paralysis (AFP) among children aged $<15$ years $(4,5)$. Stool specimens collected from children with AFP are tested for both WPV and vaccine-derived poliovirus (VDPV) in WHOaccredited laboratories within the Global Polio Laboratory Network (GPLN). In selected locations, AFP surveillance is supplemented with environmental surveillance (testing sewage for poliovirus) (G). Testing of stool and sewage samples includes genomic sequencing to characterize poliovirus isolates; results are used to map poliovirus transmission and identify gaps in AFP surveillance. This report presents poliovirus surveillance data from 2014 and 2015, focusing on the 20 countries in the African Region and six in the Eastern Mediterranean Region that reported a WPV or circulating VDPV (cVDPV) case during 2011-2015, including Guinea, Liberia, and Sierra Leone, which were most affected by the 2014-2015 Ebola virus disease (Ebola) outbreak.

During 2015, 10 (50\%) of 20 African Region countries and all six Eastern Mediterranean Region countries met both national AFP surveillance quality indicators.* To complete and certify polio eradication, surveillance gaps must be identified and surveillance activities further strengthened, including supervision, monitoring, and specimen collection and handling.

\section{Acute Flaccid Paralysis Surveillance}

In the African Region, the number of reported AFP cases increased from 22,447 in 2014 to 26,238 in 2015. The number of WPV type 1 (WPV1) cases identified decreased from 17 in four countries in 2014 to zero in 2015. Date of onset of the last WPV1 case was July 24, 2014, in Nigeria. During 2014,

\footnotetext{
* National nonpolio AFP rates of $\geq 2$ per 100,000 persons aged $<15$ years per year and $\geq 80 \%$ of AFP cases with adequate stool specimens.
}

a total of $34 \mathrm{cVDPV}$ cases (33 cVDPV type 2 [cVDPV2] and one cVDPV type 1 [cVDPV1]) were identified in four countries. During 2015, a total of 18 cVDPV cases (eight cVDPV2 and $10 \mathrm{cVDPV} 1$ ) were identified in three countries (Table 1).

In the Eastern Mediterranean Region, 12,546 AFP cases were reported in 2014 and 13,171 in 2015. The number of WPV1 cases identified declined from 342 in five countries in 2014 to 74 in the two countries with endemic polio in 2015 (20 in Afghanistan and 54 in Pakistan). During both 2014 and 2015, most of the WPV1 cases (89\% and 73\%, respectively) and all cVDPV2 cases in the region were identified in Pakistan (Table 1).

The quality of AFP surveillance is measured by two principal indicators. The first is the nonpolio AFP rate, (i.e., the number of nonpolio AFP cases per 100,000 children aged $<15$ years per year). A nonpolio AFP rate $\geq 2$ is considered sufficiently sensitive to identify WPV or cVDPV cases if poliovirus is circulating (5). The second indicator is the collection of adequate stool specimens from $\geq 80 \%$ of AFP cases, indicating surveillance can effectively identify WPV and VDPV among individuals with AFP (5). Adequacy is defined as the collection of two stool specimens $\geq 24$ hours apart, within 14 days of paralysis onset, and arrival at a WHO-accredited laboratory in "good" condition. ${ }^{\dagger}$

Among the 20 countries evaluated in the African region, 13 $(65 \%)$ and $10(50 \%)$ met both national indicators in 2014 and 2015 , respectively. Among the three countries affected by the Ebola outbreak, Guinea and Liberia did not meet one of the national indicators and Sierra Leone did not meet either indicator in 2015. All six Eastern Mediterranean Region countries examined met both indicators in 2014 and 2015 (Table 1). However, national-level surveillance indicators masked differences in surveillance performance at subnational levels (Figure).

\section{Environmental Surveillance}

Testing of sewage samples supplements AFP surveillance by identifying poliovirus transmission that might occur in the absence of detected AFP cases (๑). Environmental

\footnotetext{
$\dagger$ Reverse cold chain maintained and received without leakage or desiccation at a WHO-accredited laboratory. Reverse cold chain is maintained when stool specimens are stored immediately after collection at $4-8^{\circ} \mathrm{C}\left(32-39^{\circ} \mathrm{F}\right)$, frozen at $-20^{\circ} \mathrm{C}\left(-4^{\circ} \mathrm{F}\right)$ when received for processing, and shipped to a WHO-accredited laboratory in dry ice or cold packs. Freezing of specimens is unnecessary if specimens can be received at a WHO-accredited laboratory within 72 hours of collection.
} 
TABLE 1. National and subnational acute flaccid paralysis (AFP) surveillance indicators and number of confirmed wild poliovirus (WPV) and circulating vaccine-derived poliovirus (cVDPV) cases, by country, for all countries that had poliovirus transmission during 2011-2015 or were affected by the Ebola outbreak in West Africa within the World Health Organization (WHO) African Region and Eastern Mediterranean Region, 2014 and $2015^{*}$

\begin{tabular}{|c|c|c|c|c|c|c|c|c|}
\hline WHO region/country & AFP cases & $\begin{array}{c}\text { Regional/ } \\
\text { national } \\
\text { NPAFP rate }^{\dagger}\end{array}$ & $\begin{array}{c}\text { Subnational } \\
\text { areas with } \\
\text { NPAFP rate } \geq 2^{\S} \\
(\%)\end{array}$ & $\begin{array}{l}\text { Regional/ } \\
\text { national AFP } \\
\text { cases with } \\
\text { adequate } \\
\text { specimens } \\
\text { (\%) }\end{array}$ & $\begin{array}{l}\text { Subnational } \\
\text { areas with } \\
\geq 80 \% \text { adequate } \\
\text { specimens } \\
\text { (\%) }\end{array}$ & $\begin{array}{c}\text { Population } \\
\text { in areas } \\
\text { meeting both } \\
\text { indicators** } \\
(\%)\end{array}$ & $\begin{array}{l}\text { Confirmed } \\
\text { WPV cases* }\end{array}$ & $\begin{array}{c}\text { Confirmed } \\
\text { cVDPV cases* }{ }^{*}+\dagger\end{array}$ \\
\hline \multicolumn{9}{|l|}{2014} \\
\hline AFR & 22,447 & 5.4 & NA & 89 & NA & NA & 17 & 34 \\
\hline Angola & 321 & 3.0 & 100 & 93 & 94 & 97 & - & - \\
\hline Cameroon & 846 & 7.5 & 100 & 71 & 20 & 25 & 5 & - \\
\hline$C A R^{\S \S}$ & 89 & 4.3 & 71 & 76 & 57 & 37 & - & - \\
\hline Chad & 393 & 6.0 & 94 & 86 & 72 & 72 & - & - \\
\hline Côte d'Ivoire & 394 & 4.6 & 89 & 86 & 61 & 74 & - & - \\
\hline DRC & 1,831 & 5.4 & 100 & 82 & 82 & 76 & - & - \\
\hline Equatorial Guinea & 32 & 8.0 & 86 & 16 & 0 & 0 & 5 & - \\
\hline Ethiopiaß§ & 1,198 & 2.9 & 82 & 76 & 27 & 27 & 1 & - \\
\hline Gabon & 42 & 4.9 & 70 & 29 & 10 & 0 & - & - \\
\hline Guinea & 146 & 2.9 & 75 & 88 & 88 & 53 & - & 1 \\
\hline Kenya & 723 & 3.7 & 100 & 88 & 100 & 100 & - & - \\
\hline Liberia & 23 & 1.2 & 60 & 96 & 87 & 31 & - & - \\
\hline Madagascar & 421 & 4.2 & 82 & 85 & 55 & 63 & - & 1 \\
\hline Mali & 236 & 3.2 & 89 & 89 & 67 & 91 & - & - \\
\hline Mozambique & 317 & 2.5 & 90 & 88 & 80 & 85 & - & - \\
\hline Niger§§ & 249 & 2.5 & 86 & 71 & 14 & 14 & - & - \\
\hline Nigeria & 10,506 & 13.1 & 100 & 97 & 100 & 100 & 6 & 30 \\
\hline Republic of the Congo & 114 & 5.1 & 100 & 87 & 73 & 73 & - & - \\
\hline Sierra Leone & 72 & 2.8 & 75 & 96 & 100 & 79 & - & - \\
\hline South Sudan & 321 & 6.4 & 70 & 89 & 80 & 64 & - & 2 \\
\hline EMR & 12,546 & 6.0 & NA & 91 & NA & NA & 342 & 22 \\
\hline Afghanistan & 2,421 & 16.7 & 100 & 92 & 97 & 99 & 28 & - \\
\hline Iraq & 590 & 4.3 & 84 & 89 & 79 & 70 & 2 & - \\
\hline Pakistan & 5,369 & 8.1 & 88 & 88 & 100 & 99 & 306 & 22 \\
\hline Somalia & 420 & 8.1 & 100 & 97 & 95 & 99 & 5 & - \\
\hline Syrian Arab Republic & 306 & 4.0 & 93 & 82 & 71 & 58 & 1 & - \\
\hline Yemen & 578 & 5.8 & 100 & 95 & 100 & 100 & - & - \\
\hline
\end{tabular}

See table footnotes on next page.

surveillance collection sites increased within Afghanistan, Nigeria, and Pakistan from 21 at the end of 2011 to 83 as of March 2015. Overall, environmental surveillance is conducted in 34 countries without recent active WPV transmission, including nine on the African continent.

In Nigeria, sampling is currently conducted at 43 sites in 10 states and the Federal Capital Territory. No WPV has been isolated since May 2014 when WPV1 was isolated from one sample in Kaduna. Continued transmission of cVDPV2 that emerged in Nigeria in 2005 and of cVDPV2 imported from Chad in 2013 was documented from samples collected in six states in 2014 and only Kaduna in 2015. Environmental sampling in Afghanistan is conducted at 14 sites in five WPV high risk provinces. WPV1 was detected in samples collected in Helmand, Kandahar, and Nangarhar in 2014 and in all five provinces in 2015. In Pakistan, sampling is conducted at 40 sites in five provinces/ regions. The proportion of samples positive for WPV1 decreased from $35 \%$ in 2014 to $20 \%$ in 2015 . WPV1 was detected in all five provinces/regions in both years.

\section{Global Polio Laboratory Network}

The GPLN comprises $146 \mathrm{WHO}$-accredited poliovirus laboratories in all WHO regions. GPLN member laboratories follow standardized protocols to 1) isolate and identify poliovirus, 2) conduct intratypic differentiation to identify WPV or screen for Sabin-like poliovirus (isolates that display $\leq 1 \%$ nucleotide sequence difference from the parental vaccine strain $[\leq 0.6 \%$ for type 2$]$ ) and VDPV (7), and 3) conduct genomic sequencing. Sequencing results are used to monitor pathways of poliovirus transmission by comparing the nucleotide sequence of the coding region for one of the viral capsid proteins (VP1) of poliovirus isolates. Genomic sequencing of an isolate with $\geq 1.5 \%$ nucleotide divergence in the VP1coding region from previously identified poliovirus isolates (i.e., an "orphan" virus), indicates prolonged undetected circulation and gaps in AFP surveillance. 
TABLE 1. (Continued) National and subnational acute flaccid paralysis (AFP) surveillance indicators and number of confirmed wild poliovirus (WPV) and circulating vaccine-derived poliovirus (cVDPV) cases, by country, for all countries that had poliovirus transmission during $2011-2015$ or were affected by the Ebola outbreak in West Africa within the World Health Organization (WHO) African Region and Eastern Mediterranean Region, 2014 and $2015^{*}$

\begin{tabular}{|c|c|c|c|c|c|c|c|c|}
\hline WHO region/country & AFP cases & $\begin{array}{c}\text { Regional/ } \\
\text { national } \\
\text { NPAFP rate }^{\dagger}\end{array}$ & $\begin{array}{c}\text { Subnational } \\
\text { areas with } \\
\text { NPAFP rate } \geq 2^{\S} \\
\text { (\%) }\end{array}$ & $\begin{array}{l}\text { Regional/ } \\
\text { national AFP } \\
\text { cases with } \\
\text { adequate } \\
\text { specimens } \\
(\%)\end{array}$ & $\begin{array}{l}\text { Subnational } \\
\text { areas with } \\
\geq 80 \% \text { adequate } \\
\text { specimens } \\
\text { (\%) }\end{array}$ & $\begin{array}{l}\text { Population } \\
\text { in areas } \\
\text { meeting both } \\
\text { indicators** } \\
(\%)\end{array}$ & $\begin{array}{l}\text { Confirmed } \\
\text { WPV cases* }\end{array}$ & $\begin{array}{l}\text { Confirmed } \\
\text { cVDPV cases }{ }^{*}, t\end{array}$ \\
\hline \multicolumn{9}{|l|}{2015} \\
\hline AFR & 26,238 & 6.2 & NA & 90 & NA & NA & 0 & 18 \\
\hline Angola & 458 & 4.3 & 100 & 96 & 100 & 100 & - & - \\
\hline Cameroon & 618 & 5.6 & 100 & 83 & 80 & 67 & - & - \\
\hline CAR & 81 & 3.9 & 71 & 80 & 43 & 34 & - & - \\
\hline Chad & 435 & 6.6 & 100 & 87 & 78 & 87 & - & - \\
\hline Côte d'Ivoire & 353 & 4.0 & 94 & 90 & 89 & 94 & - & - \\
\hline $\mathrm{DRC} \S \S$ & 2,089 & 5.9 & 100 & 74 & 9 & 6 & - & - \\
\hline Equatorial Guinea & 11 & 3.6 & 43 & 36 & 0 & 0 & - & - \\
\hline Ethiopia§§ & 1,179 & 2.8 & 82 & 76 & 45 & 29 & - & - \\
\hline Gabon $§ \S$ & 61 & 8.9 & 100 & 33 & 0 & 0 & - & - \\
\hline Guinea $§ \S$ & 143 & 2.7 & 63 & 77 & 38 & 26 & - & 7 \\
\hline Kenya & 624 & 3.2 & 100 & 85 & 75 & 83 & - & - \\
\hline Liberia & 22 & 1.2 & 60 & 95 & 60 & 44 & - & - \\
\hline Madagascar & 692 & 6.4 & 100 & 60 & 14 & 23 & - & 10 \\
\hline Mali & 247 & 3.2 & 78 & 84 & 67 & 79 & - & - \\
\hline Mozambique & 323 & 2.6 & 100 & 80 & 60 & 57 & - & - \\
\hline Niger§§ & 222 & 2.3 & 71 & 59 & 0 & 0 & - & - \\
\hline Nigeria & 13,960 & 17.1 & 100 & 98 & 100 & 100 & - & 1 \\
\hline Republic of the Congo ${ }^{\S \S}$ & 117 & 5.3 & 100 & 78 & 45 & 29 & - & - \\
\hline Sierra Leone & 38 & 1.4 & 25 & 79 & 25 & 23 & - & - \\
\hline South Sudan & 329 & 6.5 & 100 & 94 & 90 & 90 & - & - \\
\hline EMR & 13,171 & 6.4 & NA & 90 & NA & NA & 74 & 2 \\
\hline Afghanistan & 2,738 & 18.9 & 100 & 93 & 94 & 94 & 20 & - \\
\hline Iraq & 520 & 3.7 & 84 & 82 & 58 & 49 & - & - \\
\hline Pakistan & 5,770 & 9.2 & 100 & 88 & 88 & 99 & 54 & 2 \\
\hline Somalia & 281 & 5.4 & 100 & 96 & 100 & 100 & - & - \\
\hline Syrian Arab Republic & 235 & 3.0 & 57 & 86 & 71 & 43 & - & - \\
\hline Yemen & 539 & 5.4 & 100 & 91 & 91 & 95 & - & - \\
\hline
\end{tabular}

Abbreviations: AFR = African Region; CAR = Central African Republic; DRC = Democratic Republic of the Congo; Ebola = Ebola virus disease; EMR= Eastern Mediterranean Region; NA = not available; NPAFP = nonpolio AFP.

${ }^{*}$ Data as of February 22, 2016.

† Per 100,000 persons aged $<15$ years per year.

$\S$ For all subnational areas regardless of population size.

" Standard WHO target is adequate stool specimen collection from $\geq 80 \%$ of AFP cases, assessed by timeliness and condition. In this analysis, timeliness was defined as two specimens collected $\geq 24$ hours apart ( $\geq 1$ calendar day in this data set), and both within 14 days of paralysis onset. Condition was defined as specimens arriving in good condition (reverse cold chain maintained and received without leakage or desiccation) in a WHO-accredited laboratory.

** For all subnational areas regardless of population size. The two indicators are NPAFP rate of $\geq 2$ per 100,000 persons aged $<15$ years per year and $\geq 80 \%$ of AFP cases with adequate stool specimens.

${ }^{+\dagger}$ cVDPV was associated with two or more cases of AFP with genetically linked VDPVs. Guidelines for classification of cVDPV changed in 2015 and can be found at http://www.polioeradication.org/Portals/0/Document/Resources/VDPV_ReportingClassification.pdf.

$\S \S$ Stool specimen adequacy dropped to $<80 \%$ when stool condition was included with timeliness. Timeliness was defined as two specimens collected $\geq 24$ hours apart ( $\geq 1$ calendar day in this dataset), and both within 14 days of paralysis onset. Condition was defined as specimens arriving in good condition (reverse cold chain maintained and received without leakage or desiccation) in a WHO-accredited laboratory.

१ๆ The NPAFP rate for Syria is artificially low because of displaced populations and the lack of official data from areas not under government control.

To meet standard laboratory timeliness indicators for stool specimen processing, laboratories should report $\geq 80 \%$ of poliovirus isolation results within 14 days of specimen receipt, $\geq 80 \%$ of intratypic differentiation results within 7 days of isolate receipt, and $\geq 80 \%$ of sequencing results within 7 days of identifying isolate intratype. The standard programmatic indicator combining field and laboratory performance is to report intratypic differentiation results for $\geq 80 \%$ of isolates within 60 days of paralysis onset for AFP cases. This indicator takes into account the interval from paralysis onset to specimen testing (the Eastern Mediterranean Region uses a 45-day time frame). The accuracy and quality of testing at GPLN laboratories is monitored through an annual accreditation program of onsite reviews and proficiency testing.

GPLN laboratories met timeliness indicators for poliovirus isolation in all regions for both years except the Western Pacific 
FIGURE. Combined performance indicators for the quality of acute flaccid paralysis (AFP) surveillance* in subnational areas (states and provinces) of 26 countries that had poliovirus transmission during 2011-2015 or were affected by the Ebola outbreak in West Africa during 2014-2015 - World Health Organization African and Eastern Mediterranean Regions, $2015^{\dagger}$

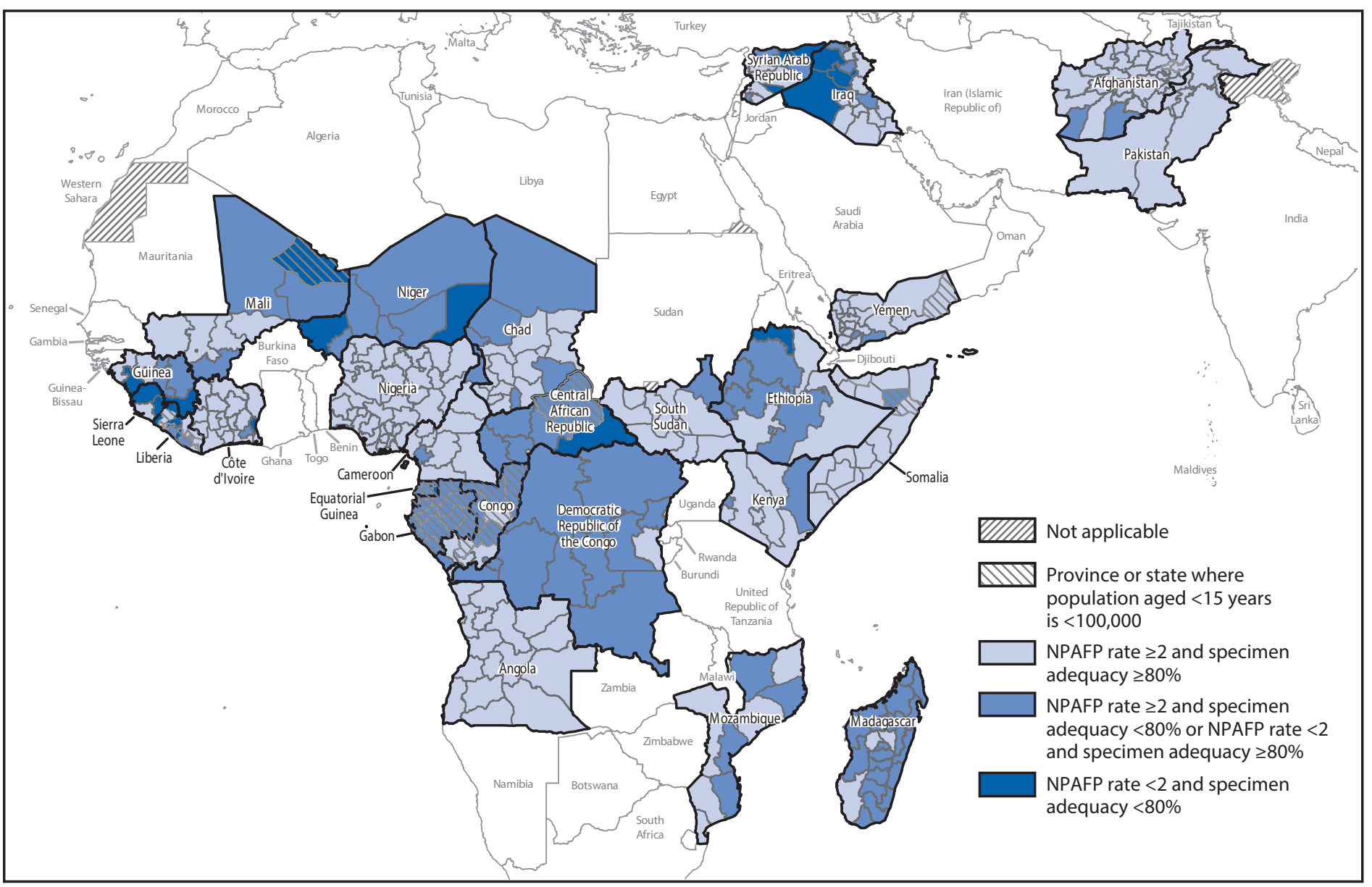

Abbreviations: Ebola = Ebola virus disease; NPAFP = nonpolio acute flaccid paralysis.

* The Global Polio Eradication Initiative has set the following targets for countries with current or recent wild poliovirus transmission and their states/provinces: 1) NPAFP rate of $\geq 2$ cases per 100,000 persons aged $<15$ years per year, and 2 ) adequate stool specimen collection from $\geq 80 \%$ of AFP cases, with specimen adequacy assessed by timeliness and condition. Timeliness was defined as two specimens collected $\geq 24$ hours apart ( $\geq 1$ calendar day in this dataset) and both within 14 days of paralysis onset. Condition was defined as specimens arriving in good condition (reverse cold chain maintained and received without leakage or desiccation) at a World Health Organization-accredited laboratory.

† Data are for AFP cases with onset during 2015, reported as of February 22, 2016.

Region in 2014 and the European Region in 2015 (Table 2). The overall timeliness indicator for onset to intratypic differentiation results was met in all regions in both years except the European Region in 2015. As of March 5, 2016, the GPLN had tested 203,698 stool specimens in 2014 and 192,250 in 2015. WPV1 was isolated from 412 AFP case samples in 2014 and from 74 in 2015. In addition, cVDPV was detected from 80 AFP case samples in 2014 and 32 in 2015.

For the first time since 2005, the majority of cVDPV cases detected globally in 2015 were caused by Type 1 . Among the $31 \mathrm{cVDPV}$ cases identified, 19 (61\%) occurred as part of type 1 outbreaks in Laos (7), Madagascar (10) and Ukraine (2); the remaining cVDPV cases were type 2 (Guinea [7], Myanmar [2], Nigeria [1], and Pakistan [2]).
Genetic diversity declined among WPV1 isolates in 2015. In 2014, West Africa B1 (WEAF-B1) and South Asia (SOAS) were the only WPV1 genotypes circulating globally. Although WEAF-B1 genotype was detected in five countries in 2014, the only genotype detected in 2015 was SOAS from Afghanistan and Pakistan.

Sequence analysis continues to indicate that, as in 2014, WPV1 and cVDPV cases were likely missed by AFP surveillance in 2015. Orphan WPV1 isolates were associated with six of 54 WPV 1 cases reported from Pakistan and two of 20 WPV1 cases reported in Afghanistan. Orphan cVDPV viruses were also isolated from stool specimens of AFP cases in Guinea, Laos, Madagascar, and Ukraine. 
TABLE 2. Number of poliovirus (PV) isolates from stool specimens of persons with acute flaccid paralysis and timing of results, by World Health Organization (WHO) region, $2014^{*}$ and $2015^{*}$

\begin{tabular}{|c|c|c|c|c|c|c|c|}
\hline \multirow[b]{2}{*}{ WHO Region } & \multirow[b]{2}{*}{ No. of specimens } & \multicolumn{3}{|c|}{ No. of poliovirus isolates } & \multirow{2}{*}{$\begin{array}{l}(\%) \\
\text { poliovirus isolation } \\
\text { results on time }\end{array}$} & \multirow{2}{*}{$\begin{array}{c}(\%) \\
\text { ITD results } \\
\text { within } 7 \text { days } \\
\end{array}$} & \multirow{2}{*}{$\begin{array}{c}(\%) \\
\text { ITD results } \\
\text { within } 60 \text { days }^{\dagger+} \\
\end{array}$} \\
\hline & & Wild & Sabin $^{\dagger}$ & cVDPV§ & & & \\
\hline \multicolumn{8}{|l|}{2014} \\
\hline African & 45,856 & 83 & 4,038 & 37 & 92 & 86 & 92 \\
\hline Americas & 1,675 & - & 39 & - & 83 & 100 & 94 \\
\hline Eastern Mediterranean & 23,552 & 329 & 809 & 27 & 98 & 95 & 97 \\
\hline European & 3,224 & - & 26 & 2 & 99 & NA & 82 \\
\hline South-East Asia & 115,539 & - & 2,785 & 3 & 97 & 90 & 98 \\
\hline Western Pacific & 13,852 & - & 352 & 11 & 78 & 96 & 81 \\
\hline Total ${ }^{\S \S}$ & 203,698 & 412 & 8,049 & 80 & 91 & 93 & 91 \\
\hline \multicolumn{8}{|l|}{2015} \\
\hline African & 50,960 & - & 3,579 & 17 & 82 & 79 & 95 \\
\hline Americas & 1,698 & - & 44 & - & 84 & 100 & 100 \\
\hline Eastern Mediterranean & 25,827 & 74 & 951 & 2 & 93 & 99 & 95 \\
\hline European & 3,655 & - & 106 & 4 & 63 & 93 & 70 \\
\hline South-East Asia & 96,783 & - & 3,335 & 2 & 97 & 86 & 98 \\
\hline Western Pacific & 13,327 & - & 194 & 7 & 96 & 98 & 86 \\
\hline Total§§ & 192,250 & 74 & 8,209 & 32 & 86 & 92 & 91 \\
\hline
\end{tabular}

Abbreviations: $\mathrm{cVDPV}=$ circulating vaccine-derived poliovirus; ITD = intratypic differentiation; $\mathrm{NA}=$ not available.

* Data as of April 1, 2015 and March 5, 2016, respectively.

† Either concordant Sabin-like results in ITD test and VDPV screening, or $\leq 1 \%$ VP1 nucleotide sequence difference compared with Sabin vaccine virus ( $\leq 0.6 \%$ for type 2).

$\S$ For PV types 1 and 3, 10 or more VP1 nucleotide differences from the respective PV; for PV type 2, six or more VP1 nucleotide differences from Sabin type 2 PV.

" Results reported within 14 days for laboratories in the following WHO regions: African, Americas, Eastern Mediterranean, South-East Asia, and Western Pacific. Results reported within 28 days for the European Region.

** Results of ITD reported within 7 days of receipt of specimen. As EURO performance might be underestimated because of data entry issues, it has been excluded from analysis.

${ }^{\dagger+}$ Results reported within 60 days of paralysis onset for all WHO regions except Eastern Mediterranean Region, which reported within 45 days of paralysis onset.

$\S \S$ For the last two indicators, total represents mean (in \%) of regions' performance.

\section{Discussion}

No WPV transmission was identified on the African continent in 2015. Certification of polio-free status requires at least 3 years of timely and sensitive surveillance (8). However, AFP surveillance indicators were not met in half of the 20 African Region countries examined. Rapid strengthening of AFP surveillance is critical to ensure timely polio-free certification of the region. This includes improving timeliness for stool collection and ensuring appropriate transport of specimens. Specimen condition was a particular concern for the Democratic Republic of the Congo, Equatorial Guinea, Ethiopia, Gabon, Niger, and the Republic of the Congo and was a factor in six countries that did not attain $\geq 80 \%$ specimen adequacy in 2015. Urgent efforts are also needed to improve AFP surveillance in Guinea, Liberia, and Sierra Leone, where health systems were severely disrupted by the Ebola outbreak (9). During 2013-2015, nonpolio AFP rates and stool specimen adequacy (i.e., timeliness and condition) declined in all three countries (10). Furthermore, specimen shipping and testing was suspended during portions of 2014-2015. Efforts are also needed to improve immunization services in these three countries. Since 2014, eight cVDPV2 cases have been detected in Guinea; cVDPV emergence is the result of low population immunity to poliovirus. The WHO African Regional Office is collaborating with eight high-risk countries on an initiative to strengthen AFP surveillance.

The national AFP surveillance quality indicators continued to be met in all six Eastern Mediterranean Region countries, including Afghanistan and Pakistan in 2015, where $94 \%$ and $99 \%$ of the population aged $<15$ years, respectively, lived in areas meeting both indicators. Nonetheless, detection of orphan viruses and environmental surveillance findings indicate continued gaps in AFP surveillance in both countries.

The findings in this report are subject to at least two limitations. First, the surveillance indicators do not fully reflect security-related issues, issues associated with mobile and difficult to access populations, or other factors that affect surveillance performance. For example, in Iraq and the Syrian Arab Republic, population movements related to conflict make interpretation of AFP surveillance indicators difficult. Second, high nonpolio AFP rates do not necessarily imply sensitive surveillance, because a proportion of reported AFP cases might not be true AFP cases and not all true AFP cases might be reported. Because AFP cases in this report are considered true AFP cases, findings are presumed to accurately depict surveillance activities in each country. Supervision and monitoring of AFP surveillance can help ensure that all true AFP cases are identified, reported, and investigated appropriately. 
As the number of reported polio cases declines, sensitive AFP surveillance becomes increasingly critical, and environmental surveillance will continue to be an important supplement to AFP surveillance. The risk for WPV and cVDPV importation, and cVDPV emergence exists even in countries within poliofree regions. To promptly identify and respond to all cases of polio, surveillance performance must be continuously assessed and quality must be maintained globally.

\section{Acknowledgments}

Situational Awareness Branch, Division of Emergency Operations, CDC; Qi Chen, Beth Henderson, Jan Iber, Division of Viral Diseases, CDC; Paul Chenoweth, Ajay Goel, Polio Eradication Department, World Health Organization (WHO); Humayun Asghar, Evgeniy Gavrilin, Varja Grabovac, Nicksy Gumede-Moeletsi, Sirima Pattamadilok, Fem Paladin, Gloria Rey-Benito, WHO GPLN laboratories, WHO Global Polio Laboratory Network.

${ }^{1}$ Global Immunization Division, CDC; ${ }^{2}$ Polio Eradication Department, World Health Organization, Geneva, Switzerland; ${ }^{3}$ Division of Viral Diseases, CDC.

Corresponding author: Cynthia Snider, csnider@cdc.gov, 404-718-6328.

\section{References}

1. World Health Organization. WHO removes Nigeria from polio-endemic list. Geneva, Switzerland: World Health Organization; 2015. http:// www.who.int/mediacentre/news/releases/2015/nigeria-polio/en/

2. Global Polio Eradication Initiative. Key countries. Geneva, Switzerland: Global Polio Eradication Initiative, World Health Organization; 2016. http://www.polioeradication.org/Keycountries.aspx

3. Moturi EK, Porter KA, Wassilak SGF, et al. Progress toward polio eradication-worldwide, 2013-2014. MMWR Morb Mortal Wkly Rep 2014;63:468-72.

4. Levitt A, Diop OM, Tangermann RH, et al. Surveillance systems to track progress toward global polio eradication-worldwide, 2012-2013. MMWR Morb Mortal Wkly Rep 2014;63:356-61.

5. World Health Organization. WHO-recommended surveillance standard of poliomyelitis. Geneva, Switzerland: World Health Organization; 2015. http://www.who.int/immunization/monitoring_surveillance/burden/ vpd/surveillance_type/active/poliomyelitis_standards/en/

6. Asghar H, Diop OM, Weldegebriel G, et al. Environmental surveillance for polioviruses in the Global Polio Eradication Initiative. J Infect Dis 2014;210:S294-303. http://dx.doi.org/10.1093/infdis/jiu384

7. Kilpatrick DR, Yang CF, Ching K, et al. Rapid group-, serotype-, and vaccine strain-specific identification of poliovirus isolates by real-time reverse transcription-PCR using degenerate primers and probes containing deoxyinosine residues. J Clin Microbiol 2009;47:1939-41. http://dx.doi.org/10.1128/JCM.00702-09

\section{Summary}

What is already known about this topic?

Surveillance is a cornerstone of polio eradication efforts. Acute flaccid paralysis (AFP) surveillance is the primary means of poliovirus detection, supplemented in selected countries by environmental surveillance. The Global Polio Laboratory Network facilitates laboratory identification of polioviruses and genomic analysis to track poliovirus spread.

What is added by this report?

There was no evidence of wild poliovirus circulation on the African continent in 2015. AFP surveillance indicators were examined in the World Health Organization African and Eastern Mediterranean Region countries that reported polio since 2011, or experienced the recent Ebola virus disease (Ebola) outbreak. The proportion that met both indicators in the African Region countries declined from 2014 to 2015. All Eastern Mediterranean Region countries met both indicators. Surveillance gaps continued at subnational levels. In Ebolaaffected countries, AFP surveillance quality weakened at national and subnational levels.

What are the implications for public health practice?

Rapid improvements in AFP surveillance are needed in African Region countries to ensure timely polio-free certification. Sensitive and timely surveillance becomes more critical as polio cases decline. Gaps in surveillance quality must be identified and resolved, especially subnationally. As long as polioviruses circulate in any country, all countries remain at risk.

8. World Health Organization. Report of the 1st meeting of the global commission for the certification of the eradication of poliomyelitis. Geneva, Switzerland: World Health Organization; 1995. http://www. polioeradication.org/Portals/0/Document/Resources/1st $\% 20$ Global\%20CertCom\%20Meeting\%20Report\%201996.PDF

9. Kieny MP, Dovlo D. Beyond ebola: a new agenda for resilient health systems. Lancet 2015;385:91-2. http://dx.doi.org/10.1016/ S0140-6736(14)62479-X

10. Porter KA, Diop OM, Burns CC, Tangermann RH, Wassilak SG. Tracking progress toward polio eradication-worldwide, 2013-2014. MMWR Morb Mortal Wkly Rep 2015;64:415-20. 